\title{
Ventilator-Associated Pneumonia in Pediatric Traumatic Brain Injury
}

\author{
Mitchell Hamele,' Chris Stockmann,, Meghan Cirulis, Jay Riva-Cambrin,2 \\ Ryan Metzger, ${ }^{3}$ Tellen D. Bennett, ${ }^{4}$ and Susan L. Bratton ${ }^{1}$
}

\begin{abstract}
Ventilator-associated pneumonia (VAP) is a common occurrence among intubated pediatric traumatic brain injury (TBI) patients. However, little is known about the epidemiology, risk factors, and microbiology of VAP in pediatric TBI. We reviewed a cohort of 119 pediatric moderate-to-severe TBI patients and identified 42 with VAP by positive protected bronchial brush specimens. Location of intubation, severity of injury, and antibiotic administration within 2 days after injury were not associated with VAP. Most treatments for elevated intracranial pressure were associated with increased risk of VAP; however, in a multi-variable analysis barbiturate coma (hazard ratio [HR], 3.2; 95\% confidence interval [CI] 1.4-7.3), neuromuscular blockade (NMBA; HR, 3.4; 95\% CI 1.6-7.3), and use of a cooling blanket for euthermia (HR 2.4; 95\% CI 1.1-5.5) remained independently associated with VAP. Most VAP (55\%) occurred prior to hospital Day 4 and only 7\% developed VAP after Day 7. Methicillin-sensitive Staphylococcus aureus (34\%), Haemophilus influenzae (22\%), and Streptococcus pneumoniae (15\%) were the most common organisms, comprising $71 \%$ of isolated pathogens (36\% of infections were polymicrobial). Patients with VAP had significantly longer intensive care unit and hospital stays, as well as increased risk of chronic care needs after discharge, but not mortality. VAP is a common occurrence in pediatric TBI patients, and early empiric therapy for patients requiring barbiturate infusion, NMBA, or use of a cooling blanket could mitigate morbidity.
\end{abstract}

Key words: pediatric; pneumonia; traumatic brain injury

\section{Introduction}

B ETWEEN 2002 AND 2006 there were 473,947 emergency department visits, 35,136 hospitalizations, and 2174 deaths related to traumatic brain injury (TBI) in patients younger than 15 years old in the United States. ${ }^{1}$ TBI is a contributing factor in $30 \%$ of injury-related deaths in all age groups. ${ }^{1}$ Ventilator-associated pneumonia (VAP) is a common occurrence among intubated children with TBI, with $6 \%$ to $40 \%$ of children intubated for at least 2 days developing VAP. ${ }^{2-5}$ VAP has been associated with prolonged mechanical ventilation, increased intensive care unit (ICU) and hospital length of stay, and in some studies, greater mortality. ${ }^{5-13}$

Despite the frequency of VAP complication pediatric TBI, little is known about the risk factors for and microbiology of VAP in these patients. This information could guide efforts to prevent VAP, as well as guide appropriate empiric therapy. Two prior reports regarding the microbiology of VAP complicating pediatric trauma victims were limited by their small sample size $(n<18){ }^{5,10}$ Given the lack of large studies, practitioners are forced to extrapolate from the epidemiology of VAP in general ICU pediatric and adult patients to guide empiric therapy and diagnostics. Known risk factors in these populations include pre-existing medical conditions, prolonged mechanical ventilation, or chronic hospitalizations, among others, and common organisms include Pseudomonas aeruginosa, Staphylococcal spp., and resistant gram-negative organisms. ${ }^{8,11,12,14-17}$ It is likely that both the risk factors and microbiology of VAP in the pediatric TBI population differ from chronically ill or hospitalized populations.

The ability to predict which intubated pediatric TBI patients are at increased risk for VAP and at what time during their hospital course they are at risk, and to target the most common pathogens for empiric therapy could improve outcomes in this population. We evaluate a cohort of intubated and mechanically-ventilated pediatric TBI patients and describe the epidemiology, risk factors and microbiology associated with VAP.

\section{Methods}

We determined the prevalence and risk factors for VAP in children with moderate-to-severe TBI (post-resuscitation Glasgow Coma Scale $[\mathrm{GCS}]$ score of $<13$ ). We retrospectively reviewed all pediatric patients admitted at a level one pediatric trauma center

${ }^{1}$ Department of Pediatrics, ${ }^{2}$ Department of Neurosurgery, ${ }^{3}$ Division of Pediatric Surgery, University of Utah School of Medicine, Salt Lake City, Utah.

${ }^{4}$ Pediatric Critical Care, University of Colorado School of Medicine, Aurora, Colorado. 
between January 2009 and January 2012 with moderate or severe TBI who received mechanical ventilation for 2 days or more after injury. Administrative data, including the local trauma database, were used to identify children admitted to the pediatric intensive care unit (PICU) after head injury. The institutional review board at the University of Utah and privacy board for Primary Children's Hospital reviewed this study and waived the need for informed consent.

Primary Children's Hospital is an American College of Surgeons-verified level I trauma center caring for injured children in a five-state catchment area. Approximately 800 trauma patients are admitted to the inpatient service, with 80 admitted to the PICU annually. All patients admitted to the PICU with severe TBI receive multi-disciplinary care, including: pediatric trauma surgery, neurosurgery, critical care, physical medicine and rehabilitation, and palliative care when indicated.

Criteria for VAP in children remain controversial. ${ }^{18}$ We defined VAP as a pulmonary process developing on or after hospital Day 2 with clinical suspicion of pneumonia based on fever, elevated or depressed white count, increased secretions, decreased pulmonary compliance or oxygenation, and required a microbiologic specimen deemed positive by Centers for Disease Control and Prevention (CDC) criteria. Microbiologic specimens were collected at the discretion of the medical team and were not obtained in patients without signs or symptoms of pneumonia. Criteria enumerated by the CDC to define a positive specimen are purulent sputum and $\geq 10^{3}$ colony-forming unit/high powered field (CFU/hpf) for protected bronchial brush specimens (PBBS) and $>10^{4}$ CFU/hpf for either tracheal aspirate (TA) or bronchiolalveolar lavage (BAL) with commensal organisms excluded. Microbiologic specimens were considered purulent if there were $\geq 25$ neutrophils and $\leq 10$ epithelial cells per low power field. Patients were considered no longer at risk of VAP 2 days after extubation, at the time of tracheostomy placement or death, whichever came first. Patients who did not have a specimen collected or who did not have a positive microbiologic specimen were categorized as not having VAP. Chest radiographic information was collected but not utilized as findings in this trauma population were very non-specific and microbiologic (largely PBBS) specimens, which are more specific, were required for diagnosis. ${ }^{8,24}$ Patients who died or developed pneumonia prior to hospital Day 2 were excluded from analysis.

Demographic and injury data were collected, including age, race, and mechanism of injury. Mechanism of injury was divided into non-accidental trauma, blunt trauma involving motorized vehicles and blunt trauma not involving motorized vehicles. Emergency medical transport team notes or emergency department records were used to identify the location of intubation. Rapid sequence intubation is part of local emergency medical service and emergency department protocols but its use was not collected as documentation was not consistent. Injury severity was evaluated using post-resuscitation GCS, chest Abbreviated Injury Scale (AIS) score, Injury Severity Score (ISS), and Rotterdam computed tomography (CT) scores which were independently assigned by a neurosurgeon blinded to patient outcome. ${ }^{19,20}$

The need for and type of intracranial pressure (ICP) monitoring device was determined based on clinical need by the treating neurosurgeon. ICP and cerebral perfusion pressure (CPP) were abstracted from the daily nurse charting. We collected data regarding administration of antibiotics in the first 2 days of hospitalization prior to the patients being at risk of VAP. We evaluated intensive care therapies used to monitor and treat intracranial hypertension, such as: administration of continuous infusions of narcotics, sedatives, barbiturates, neuromuscular blocking agents (NMBA), hypertonic saline, or vasopressors, as well as boluses of osmolar agents, such as mannitol.

We also evaluated receipt of enteral feeds or total parenteral nutrition (TPN), which also have been reported as risk factors for VAP. ${ }^{7,16}$ Source of nutrition was recorded as none, TPN, gastric or transpyloric feeds. Histamine-2 blockers or proton pump inhibitors were routinely given to patients not being fed via the stomach for prophylaxis of stress gastric ulcers. Outcomes are reported as discharge to home with no long-term care, discharge to long-term care on a rehabilitation service, skilled nursing facility or home health care, or death prior to discharge. Care guidelines for mitigating and treating intracranial hypertension that mirror recommendations from the Brain Trauma Foundation (Supplementary Fig. 1; see online supplementary material at www.liebertpub.com) were used by the clinical teams. ${ }^{21}$ The care guidelines include temperature regulation to maintain euthermia $\left(36-38^{\circ} \mathrm{C}\right)$, ICP goal $<20 \mathrm{~mm} \mathrm{Hg}$ when monitored, normocapnia (partial pressure of carbon dioxide $\left[\mathrm{PaCO}_{2}\right], 35-40 \mathrm{~mm} \mathrm{Hg}$ ), and serum sodium concentration $>140 \mathrm{mEq} / \mathrm{L}$. Once these are optimized, subsequent persistent intracranial hypertension is treated serially with sedation/analgesia, administration of NMBA (typically vecuronium $0.1 \mathrm{mg} / \mathrm{kg} / \mathrm{h}$ ), osmolar therapy with mannitol or $3 \%$ saline, an external ventricular drain or lumbar drain if feasible, barbiturate infusion with a goal of burst suppression on electroencephalography (EEG), and mild hyperventilation $\left(\mathrm{PaCO}_{2}, 30-35 \mathrm{~mm} \mathrm{Hg}\right)$.

Patients with and without VAP were compared using the MannWhitney $U$ test for continuous and categorical variables. Demographic and static risk factors were compared by group and reported as relative risk ratios RR with $95 \%$ confidence intervals (CIs) or as median values and corresponding interquartile range (IQR). Because risk of VAP increases with duration of mechanical ventilation and exposure to intensive care therapies, we used a Cox proportional hazard model adjusted for duration of mechanical ventilation and sought to identify potentially modifiable ICU risk factors for VAP. Patients were censored once they developed VAP, had a tracheostomy tube placed, were extubated, or died. The hazard ratio (HR) with $95 \%$ CI are reported.

Covariate selection for the Cox model was performed using stepwise forward and backward variable selection procedures. ${ }^{22}$ To identify ICU therapies that were independently associated with an increased hazard of VAP, a $p$ value of $<0.20$ was required for inclusion in the model during the forward selection phase. In the backward selection phase, ICU therapies were excluded from the multivariable model if they had a $p$ value $\geq 0.05$.

Additionally, time-dependent covariates were tested in the Cox proportional hazards models. These models were constructed to determine whether the effects of ICU therapies on the risk of VAP varied based on the time at which they were initiated (e.g., the 2nd versus the 10th hospital day), compared with any exposure to the ICU therapy throughout the entire duration of mechanical ventilation. These Cox proportional hazards models were developed using the survival package in R 3.0.2 (R Foundation for Statistical Computing, Vienna, Austria). ${ }^{23}$ Additional statistical analyses were performed using SPSS version 22.0 (IBM Corporation, Armonk, NY).

\section{Results}

One hundred and twenty eight trauma patients were treated in the PICU during the study period for TBI and had a post-resuscitation GCS score $<13$. Nine patients were excluded from analysis leaving 119 patients (Fig. 1). Table 1 outlines demographic and nonmodifiable injury characteristics categorized by presence or absence of VAP. Patients with VAP were significantly older, with a median age of 9 years, compared with 6 years in those without VAP. Nonwhite children and those suffering from non-accidental trauma were less likely to have VAP, compared with white children and those with accidental injuries. Seventy-nine (64\%) patients were intubated at a referring hospital, while $25 \%$ were intubated at the scene. Site of intubation was not associated with VAP. Compared with patients who did not have a PBBS specimen tested, tested patients were significantly older ( 9 years vs. 5 years) and more likely to be treated 


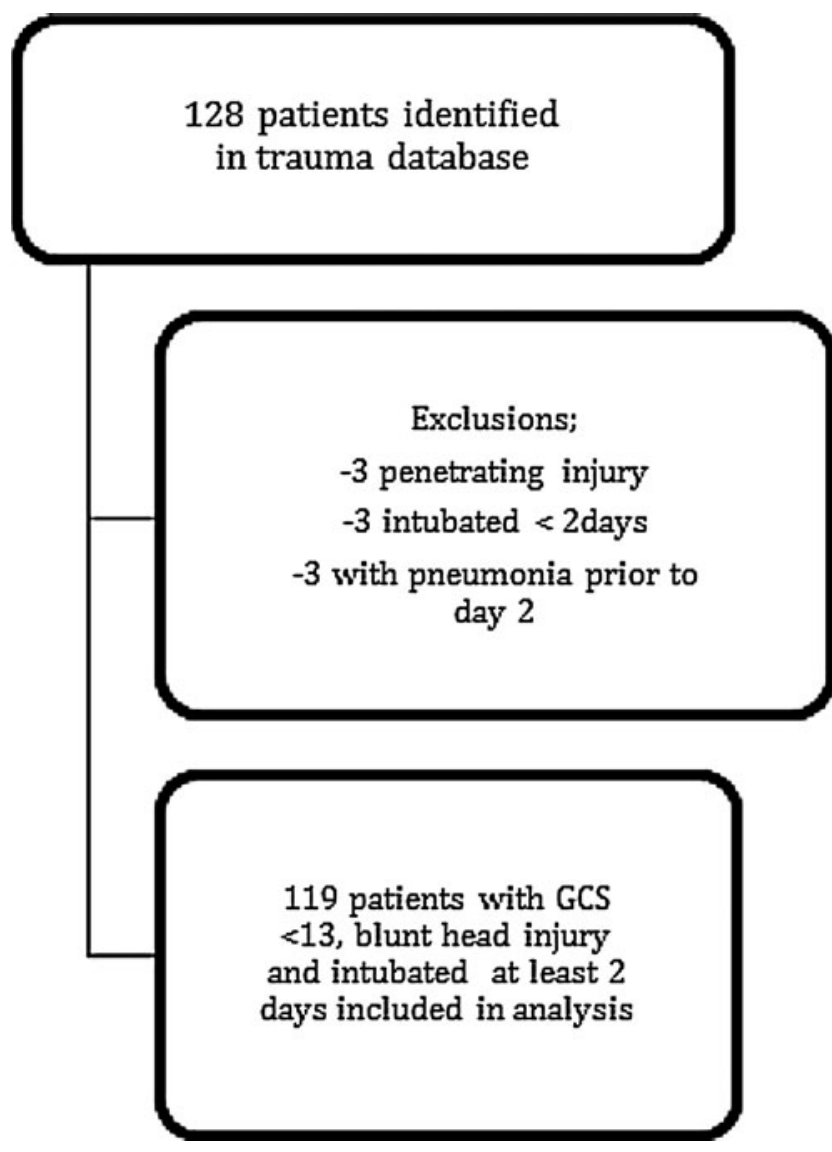

FIG. 1. Inclusion/exclusion criteria. GCS, post-resuscitation Glasgow Coma Scale score.

with therapies for intracranial hypertension (eg., NMBA [32\% vs. $12 \%$ ], $3 \%$ saline [62\% vs. $30 \%$ ], sedative [71\% vs. $54 \%$ ], and vasopressors [53\% vs. 30\%]), and to have longer median duration of mechanical ventilation (9 days vs. 3 days), longer length of stay in the hospital (21 days vs. 9 days), and lower rates of death (10\% vs. $26 \%$ ), but a greater rate of discharge to rehabilitation (64\% vs. $19 \%$ ).

Figure 2 shows the time to VAP development. Forty-two of 119 patients developed VAP (36\%), which was most frequently diagnosed on Day 2-3 after admission in 23 of $42(55 \%)$ patients. Late-onset VAP was a rare entity in our cohort, with only 3 of 42 (7\%) diagnosed with VAP after Day 7.

Table 1 also shows univariate comparisons of severity of injury measures for children with and without VAP. The study groups had similar median chest AIS, ISS, GCS, and Rotterdam head CT scores. However, patients who had an ICP monitor placed or had a single measurement of ICP $>20 \mathrm{~mm} \mathrm{Hg}$ had a more than two-fold greater risk of VAP.

Table 2 presents the unadjusted hazard ratios with $95 \%$ CI for ICU therapies, including exposure to nutrition and antibiotics after injury. Most therapies, except sedative infusions, administration of mannitol, and antibiotics, were associated with increased hazard of VAP, with the largest estimated increases associated with prolonged neuromuscular blockade, use of a cooling blanket to control fever, and barbiturate coma. No individual route of nutrition was significantly associated with hazard of VAP. When gastric and transpyloric feeding were combined, there appeared to be a trend toward protection against VAP (HR, 0.54; 95\% CI 0.27-1.09; $p=0.09$ ), compared with no enteral nutrition. Exposure to antibiotics on hospital Day 0 or 1 did not affect risk of VAP. Interestingly, receiving any antibiotic primarily active against gram negative organisms on hospital Day 0 or 1 appeared to be protective, while exposure to antibiotics with greater activity against skin commensals did not.

Table 3 presents the multi-variable model of those factors that were most strongly associated with VAP in the univariate analysis. Factors independently associated with hazard of VAP on multivariate analysis were use of a cooling blanket to prevent fever (HR 2.4), administration of prolonged neuromuscular blockage (HR, 3.4), and barbiturates for burst suppression on electroencephalography (EEG; HR, 3.2). When the independently significant factors were tested for cumulative risk for increased exposure over time, no significant increased risk with increased days of ventilation was noted. When controlled for injury severity, there was no difference in the model.

Control of fever often required the use of a cooling blanket, which leads to shivering, and use of NMBA to limit the concomitant increase in metabolic demand. Both barbiturate coma and NMBA decrease coughing and patient movement, limiting secretion clearance. Given that these three therapies are often linked, we tested the hazard of VAP for patients exposed to any of the three treatments for intracranial hypertension, which is shown as a Kaplan Meier curve (Fig. 3). By hospital Day 5, just more than half of children receiving cooling, paralysis and or barbiturates developed VAP, while only about $15 \%$ of those not receiving any of these three treatment developed pneumonia. By 10 days, $88 \%$ of children treated with any of the three treatments developed VAP, compared with $30 \%$ of those did not receive any of these treatments but remained intubated.

Fifty-nine different pathogens were isolated from 42 patients with VAP. Forty-one of $42(98 \%)$ patients had specimens collected using a protected bronchial brush. Twenty-seven (64\%) patients had one pathogen isolated while $15(36 \%)$ patients had polymicrobial infections. The majority of pathogens isolated, were not resistant organisms; methicillin-sensitive Staphylococcus aureus (MSSA; 34\%), Haemophilus influenza (22\%), and Streptococcus pneumonia (15\%) comprised $71 \%$ of isolated pathogens. Two (3\%) patients had methicillin-resistant Staphylococcus aureus (MRSA) and three $(5 \%)$ patients had potentially resistant gram-negative enteric pathogens. (Supplementary Fig. 1).

VAP was associated with significantly longer median length of mechanical ventilation, hospital stay, and ICU stay, as well as a greater than two-fold increased risk of being discharged with home health services $(n=1)$, to a rehabilitation facility $(n=25)$, or to a nursing care facility $(n=6)$, compared with home $(n=10)$ without services, but was not associated with risk of death. (Table 4).

\section{Discussion}

In this study of children with moderate-to-severe TBI treated with mechanical ventilation for 2 days or more, just over a third $(36 \%)$ were diagnosed with VAP. VAP was associated with older age and some measures of head injury severity but was not associated with site of initial intubation or severity of chest injury. Treatment for intracranial hypertension with barbiturates, NMBA, and use of a cooling blanket for euthermia was associated with large increases in VAP as days of ventilation increased. We did not find significant associations between VAP and feeding route. The majority of VAP was diagnosed in the first 5 days of hospitalization and the pathogens were largely commensal organisms of the skin 
Table 1. Demographic and Clinical Features of 119 Head-Injured Children, Comparing Those with and without Ventilator-Associated Pneumonia (VAP)

\begin{tabular}{|c|c|c|c|}
\hline & $\begin{array}{c}V A P \\
\mathrm{~N}=42(\mathrm{n}, \%)\end{array}$ & $\begin{array}{c}\text { No VAP } \\
\mathrm{N}=77(\mathrm{n}, \%)\end{array}$ & $\begin{array}{l}\text { Relative risk (95\% confidence interval) } \\
\text { or } \mathrm{p} \text { value (Mann Whitney } U \text { test })\end{array}$ \\
\hline Female & $13(29)$ & $21(27)$ & $1.1(0.7-1.9)$ \\
\hline Age (years, median IQR) & $9(6-13)$ & $6(1.5-13)$ & $p=0.03$ \\
\hline \multicolumn{4}{|l|}{ Race } \\
\hline White & $37(88)$ & $54(70)$ & 1 \\
\hline Non-white & $5(11)$ & $23(30)$ & $0.4(0.2-1.0)$ \\
\hline \multicolumn{4}{|l|}{ Mechanism of injury } \\
\hline Non-accidental trauma & $3(7)$ & $18(23)$ & 1 \\
\hline Motorized trauma & $27(64)$ & $42(54)$ & $2.7(0.9-8.1)$ \\
\hline Non-motorized trauma & $12(29)$ & $16(21)$ & $3.0(1.0-9.3)$ \\
\hline \multicolumn{4}{|l|}{ Site of intubation } \\
\hline Scene & 8 (19) & $21(26)$ & 1 \\
\hline Referring hospital & $28(67)$ & $52(65)$ & $1.0(0.7-2.4)$ \\
\hline Trauma center emergency department & $4(10)$ & $5(6)$ & $1.7(0.6-4.0)$ \\
\hline \multicolumn{4}{|l|}{ Injury severity } \\
\hline Cardiac arrest prior to admission & $4(9)$ & $8(10)$ & $0.9(0.4-2.1)$ \\
\hline Median ISS & $30(25-42)$ & $29(22-35)$ & $p=0.15$ \\
\hline ISS $>20$ & $40(95)$ & $60(78)$ & $1.2(1.0-1.4)$ \\
\hline Any chest AIS >3 & $3(7)$ & $9(12)$ & $0.6(0.3-1.9)$ \\
\hline GCS $<9$ & $34(81)$ & $61(79)$ & $1.1(0.6-2.0)$ \\
\hline Rotterdam CT score & $3(2-4)$ & $3(2-4)$ & $p=0.17$ \\
\hline Any ICP monitor & $38(90)$ & $33(44)$ & $2.1(1.4-2.8)$ \\
\hline ICP high $>20 \mathrm{~mm} \mathrm{Hg}$ & $20(48)$ & $14(18)$ & $2.5(1.4-4.6)$ \\
\hline CPP low $<46 \mathrm{~mm} \mathrm{Hg}$ & $12(29)$ & $12(16)$ & $1.6(1.0-2.6)$ \\
\hline
\end{tabular}

Motorized = blunt trauma involving motorized vehicle; non-motorized $=$ accidental blunt trauma; scene = intubation in the field by emergency medical services personnel; any ICP monitor = external ventricular or fiber optic intracranial pressure monitor. CHECK

ISS, Injury Severity Score; AIS, Abbreviated Injury Scale; GCS, post-resuscitation Glasgow Coma Scale score; CT, computed tomography; ICP, intracranial pressure; CPP, cerebral perfusion pressure.

and upper respiratory tract. Antibiotics given on hospital Day 0 or 1 did not affect VAP diagnosis. Finally, VAP complicating TBI was associated with longer ICU and hospital stays, as well as worse outcome at discharge, but not mortality.

We found that $36 \%$ of patients developed VAP. A recent study by Alharfi and colleagues ${ }^{5}$ in 180 pediatric patients with severe TBI evaluating at all hospital acquired infections (HAI) found $6 \%$ of their patients developed VAP defined by the CDC criteria. The injury severity in that cohort was similar to ours but with slightly higher overall mortality and longer median ventilator times. We suspect the difference in VAP rate is due to variability in the application and interpretation of criteria for VAP. Our institution

\section{Days to pneumonia}

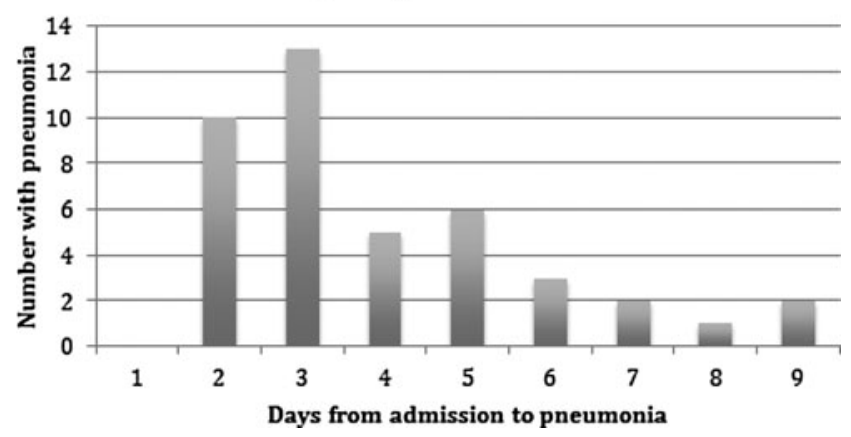

FIG. 2. Incidence of ventilator-associated pneumonia and day of mechanical ventilation. follows established VAP prevention bundles. VAP diagnosis relied on a strict definition requiring clinical suspicion by the treating team and did not include abnormal findings on chest radiography, but instead relied on microbiologic specimen meeting CDC guidelines, with $98 \%$ collected via PBBS which has been shown to be $95 \%$ specific in identifying VAP. ${ }^{24}$ Additionally, Alharfi and colleagues reported a lower rate of VAP in severe TBI that was lower than rates from three larger studies in children evaluating therapeutic hypothermia in TBI that reported VAP complicating $26 \%$ to $40 \%$ of patients regardless of treatment arm..$^{2-4}$ Many studies in adult patients report VAP rates in their severe TBI patients of $26 \%$ to $43 \%$, which are also similar to ours. ${ }^{25-28}$

Patients who developed VAP tended to be older, had higher rates of accidental trauma and were more likely to be white than those who did not. Higher risk among older patients also was observed in other studies in pediatric trauma associated VAP, although our VAP cohort was slightly younger than that in the work led by Alharfi and by Taira. ${ }^{5,10}$ Very little has been reported regarding inflicted head trauma and VAP, and the lower rate may be due to decreased use of ICP monitors secondary to open fontanelles and injury pattern and consequently less use of medications that suppress cough and inhibit the immune system to control ICP. Site of intubation was not associated with VAP. Other authors report intubation at the scene of injury or in the emergency department as a VAP risk factor. ${ }^{29}$

The majority ( $80 \%$ ) of patients who developed VAP did so early, in the first 5 days. This is similar to the work by Alharfi and colleagues who found 10 of 13 cases of VAP in their cohort developing VAP prior to one week. ${ }^{5}$ In contrast, studies by Adelson and 
Table 2. Ventilator-Associated Pneumonia (VAP) and Intensive Care Therapies

\begin{tabular}{lccc}
\hline Therapy & VAP & No VAP & $\begin{array}{c}\text { Hazard ratio } \\
(95 \% \text { CI })\end{array}$ \\
\hline Sedative infusion & $\mathrm{N}=77(\mathrm{n}, \%)$ & $1.7(0.8-3.3)$ \\
Narcotic infusion & $35(78)$ & $42(55)$ & $2.9(1.3-6.5)$ \\
$3 \%$ saline infusion & $33(73)$ & $44(57)$ & $3.4(1.8-6.4)$ \\
Mannitol bolus & $29(64)$ & $26(34)$ & $2.8(0.7-11.6)$ \\
Any vasopressor infusion & $3(7)$ & $1(1)$ & $3.2(1.7-6.0)$ \\
Targeted euthermia & $24(53)$ & $26(34)$ & $6.1(3.1-12.1)$ \\
NMBA infusion & $13(29)$ & $15(19)$ & $5.5(2.9-10.3)$ \\
Barbiturate infusion & $14(31)$ & $12(16)$ & $4.6(2.1-10.2)$ \\
Total parenteral nutrition & $4(9)$ & $1(1)$ & $1.6(0.7-3.7)$ \\
Transpyloric Feeds & $6(14)$ & $44(57)$ & $0.7(0.4-1.4)$ \\
Gastric Feeds & $18(43)$ & $15(19)$ & $0.8(0.3-2.0)$ \\
Antibiotics during days 0-1 & $5(12)$ & $47(61)$ & Relative risk ratio (95\% CI) \\
Any antibiotic & $20(48)$ & $35(45)$ & $0.7(0.4-1.2)$ \\
Skin/upper respiratory antibiotic coverage & $19(45)$ & $24(31)$ & $1.0(0.6-1.6)$ \\
Gram-negative antibiotic coverage & $5(12)$ & $2(3)$ & $0.4(0.2-1.0)$ \\
MRSA antibiotic coverage & 0 & -
\end{tabular}

All therapies administered as a continuous infusion except when noted as a bolus. Sedative infusion=any benzodiazepine or dexmetetomidine; any vasopressor infusion $=$ norepinephrine, epinephrine, or dopamine; targeted euthermia $=$ use of cooling blanket to achieve euthermia $\left(36-38^{\circ} \mathrm{C}\right)$; total parental nutrition = transpyloric feeds, and gastric feeds are prior to VAP, tracheotomy, extubation, or death; skin/upper respiratory coverage on hospital day 0 or $1=$ amoxicillin/clavulanic acid, cefazolin, cefuroxime, cephalexin, clindamycin, nafcillin, ampicillin/sulbactam; gram-negative antibiotic coverage on hospital day 0 or $1=$ ceftriaxone, gentamicin, pipercillin/tazobactam, meropenem; MRSA (methicillin-resistant Staphylococcus aureus) antibiotic coverage on hospital Day 0 or $1=$ linezolid or vancomycin.

CI, confidence interval; NMBA, neuromuscular blocking agents.

colleagues of severely brain-injured children testing if early hypothermia improved outcome reported that approximately half of VAP occurred after 1 week. ${ }^{3,4}$ Reports of VAP among ventilated general ICU patients report $50 \%$ to $58 \%$ of VAP occurring during the first week of mechanical ventilation. $6,7,9$

We evaluated several measures of both brain and total body injury severity and found inconsistent associations with VAP. No significant difference in median ISS, GCS, or Rotterdam CT scores was noted. We did find that ICP monitor placement and a single ICP measurement $>20 \mathrm{~mm} \mathrm{Hg}$ were associated with a twofold increase in risk of VAP. This suggests that patients with clinically more significant brain injury have higher risk of VAP. Alharfi and colleagues found that pediatric patients with an ICP monitor have increased rates of HAI but did not report VAP $(n=11)$ seperately. ${ }^{5}$ Others reported an ISS score $>25$ and a lower GCS score were associated with increased risk of VAP in seven patients, but these differences were not statistically significant. ${ }^{10}$ Among adults with trauma higher ISS and chest AIS scores, as well as lower post-resuscitation GCS scores, increase risk of VAP, which may be related to larger number of patients studied. $^{27}$

Table 3. Multivariable Model with Step-Wise FORWARD PROCEDURE FOR FACTORS INDEPENDENTLY AsSOCIATED WITH VAP

\begin{tabular}{lcc}
\hline Factor & $\begin{array}{c}\text { Hazard } \\
\text { ratio }\end{array}$ & $\begin{array}{c}95 \% \\
\text { confidence } \\
\text { interval }\end{array}$ \\
\hline Neuromuscular blocking agent infusion & 3.4 & $1.6-7.3$ \\
Cooling blanket to prevent fever & 2.4 & $1.1-5.5$ \\
Barbiturates for coma & 3.2 & $1.4-7.3$ \\
\hline
\end{tabular}

VAP, ventilator-associated pneumonia.
Therapies for intracranial hypertension independently associated with VAP included prolonged NMBA use, cooling blanket use for euthermia, and barbiturate coma, with just over half of children who had one or more of these treatments developing VAP by hospital Day 5 and $88 \%$ by Day 10. In adult patients, Lepelletier and colleagues ${ }^{14}$ reported barbiturate use increased risk of VAP in trauma patients. Neuromuscular blockade has been associated with VAP by some but not other researchers. ${ }^{9,15}$ We did not assess all previously reported risk factors for VAP, such as transport out of the ICU, genetic syndromes, bronchoscopy, histamine-2 (H-2) blockers, or immunodeficiency. ${ }^{8,9,11,13,15}$ However, H-2 blockers and proton pump inhibitors are used routinely in our brain-injured patients, genetic syndromes are rare, and bronchoscopy in braininjured patients is rarely performed in our unit. There is evidence that brain-injured patients are functionally immunosuppressed beyond the typical compensatory anti-inflammatory response syndrome seen in critically ill patients, but we did not assess T-cell levels or other measures of immune function directly. ${ }^{30}$

Several other ICP-lowering or CPP elevating therapies, such as narcotics, 3\% saline, and vasopressor infusions, were univariately associated with risk of VAP, but not as strongly as barbiturates, cooling to euthermia, and NMBA. Few studies have assessed the association between elevated ICP measurements and development of VAP in pediatric patients. We believe this association between VAP, NMBA, barbiturate coma, and cooling to euthermia is likely due to prevention of patient-initiated coughing and secretion clearance, as well as the immune suppressant effect of barbiturates. ${ }^{31-33}$ Limitation of the use of these therapies to only those with ICP elevation threatening herniation may be reasonable to decrease rates of VAP in this population.

We found no significant association between early initiation of nutrition by any route (TPN, gastric, or transpyloric feeding) and development of VAP. When gastric and transpyloric enteral feeding were grouped together, there was a trend towards a protective effect. Other studies in the general pediatric and adult populations 


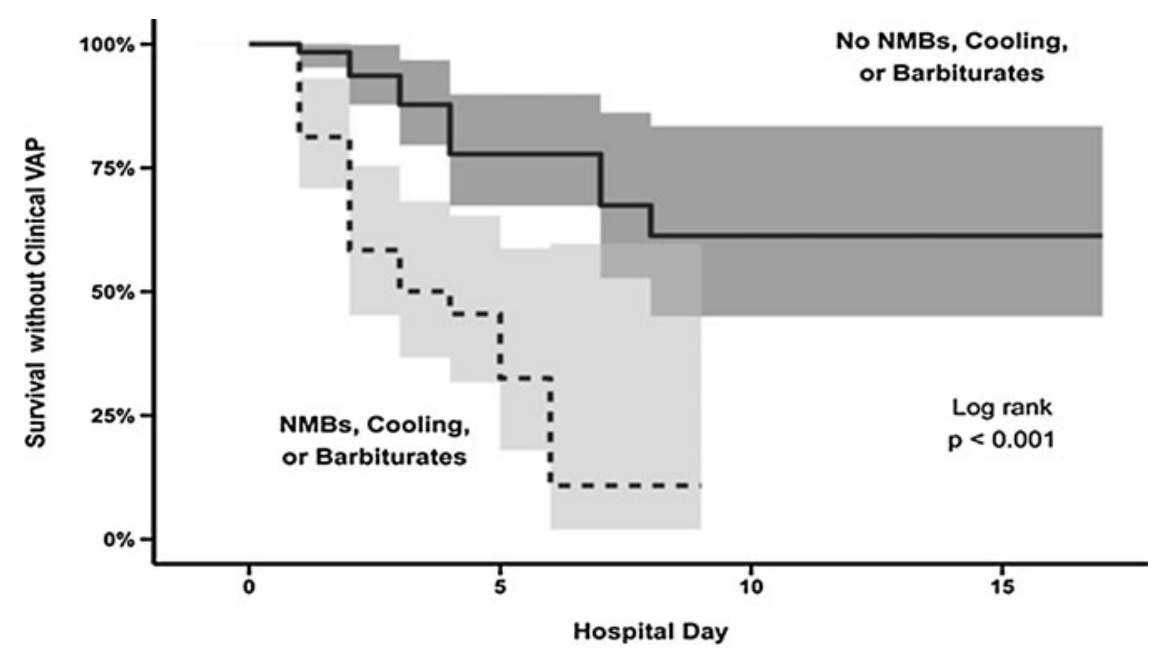

FIG. 3. Kaplan-Meier curves depicting the time to ventilator-associated pneumonia (VAP) stratified by the use of neuromuscular blocking agents, a cooling blanket for targeted euthermia, or barbiturate infusion for coma.

have been inconclusive regarding the role of gastric feeding in VAP as feeding has been found to be a risk factor, to confer protection, or not to be associated with VAP., ${ }^{7,9,13,17}$

Administration of antibiotics in the first 2 days of hospitalization did not lower risk of VAP. A study of adult TBI victims who received two "prophylactic" doses of $1500 \mathrm{mg}$ of cefuroxime showed decreased incidence of VAP. ${ }^{28}$ There was no difference in VAP rates among patients who received antibiotics on the first 2 days with anti-microbial activity against the majority of pathogens identified. Surprisingly, however, a sub-group of patients who received broader coverage for gram-negative bacteria (ceftriaxone, pipercillin/tazobactam, gentamicin, or meropenem) in the first 2 hospital days had lower risk of VAP. The 2007 Brain Trauma Foundation guidelines include a level 2 recommendation supporting peri-procedural antibiotic administration for intubation. ${ }^{34}$ Given our results, it appears that using typical prophylactic or periprocedural antibiotics, such as cefuroxime or cefazolin, is unlikely to significantly lower VAP in children.

We found that the majority of patients in our cohort were infected with common organisms of the skin and upper respiratory tractMSSA, H. influenza, and Streptococcus pneumonia - similar to the pathogen identified by Alharfi and colleauges. ${ }^{5}$ We identified very few resistant and potentially resistant bacteria, with $3 \%$ infected with MRSA and 5\% with potentially resistant gram negatives. This anti-microbial pattern differs from reports of VAP in general ICU patients where gram-negative organisms, particularly $P$. aeruginosa, comprise the majority of pathogens. ${ }^{8,9,11-13,18}$ Additionally, we found that $36 \%$ of infections were polymicrobial, similar to the work of Srinivasan and colleagues. ${ }^{9}$ This suggests that in brain-injured intubated pediatric patients, antibiotic coverage for typical, nonresistant upper respiratory tract and skin organisms, such as single therapy with a $\beta$-lactam antibiotic or cephalosporin, is likely adequate for empiric treatment, but not for prophylaxis of VAP.

Finally VAP was associated with prolonged length of mechanical ventilation, ICU stay, and hospital length of stay, consistent with previous work in both adult and pediatric brain-injured and general ICU patients. ${ }^{5-13}$ VAP was not associated with mortality but was associated with worse functional outcome among our patients who survived to discharge. Some studies have shown increased risk of mortality among patients with VAP in both the trauma and general ICU population. ${ }^{6-9,11}$ It is possible that the more significantly injured multitrauma or head-injured patients tended to die prior to developing VAP, causing the trend towards decreased risk in our population.

Our study has the limitations inherent to retrospective studies, including inability to imply causality and control for practice variation over time and between providers. However, our institution

Table 4. Outcomes among Those with and without VAP

\begin{tabular}{|c|c|c|c|}
\hline Outcomes & $V A P(\mathrm{n}=42)$ & No VAP $(\mathrm{n}=77)$ & $\begin{array}{l}R R(95 \% \text { CI) or } \mathrm{p} \text { value } \\
\text { (Mann Whitney U test })\end{array}$ \\
\hline $\begin{array}{l}\text { Ventilation duration (days) } \\
\text { Median (IQR) }\end{array}$ & $9(7-12)$ & $4(2-6)$ & $p<0.001$ \\
\hline $\begin{array}{l}\text { ICU length of stay (days) } \\
\text { Median (IQR) }\end{array}$ & $11.9(8.4-13.9)$ & $4.9(3.1-6.9)$ & $p<0.001$ \\
\hline $\begin{array}{l}\text { Hospital length of stay (days) } \\
\text { Median (IQR) }\end{array}$ & $21(16-23)$ & $9(6.5-12)$ & $p<0.001$ \\
\hline \multicolumn{4}{|l|}{ Discharge status $n(\%)$} \\
\hline Home & $9(21)$ & $31(40)$ & 1 \\
\hline $\begin{array}{l}\text { Home health services, rehabilitation } \\
\text { facility, or long term nursing care }\end{array}$ & $30(71)$ & $27(35)$ & $2.34(1.25-4.37)$ \\
\hline Death prior to discharge & $3(7)$ & $19(25)$ & $0.61(0.21-1.74)$ \\
\hline
\end{tabular}

VAP, ventilator-associated pneumonia; CI, confidence interval; IQR, interquartile range; RR, risk ratio; ICU, intensive care unit. 
follows ICP management guidelines modeled on the Brain Trauma Foundation Guidelines that remained constant over the study period. We did not use the CDC PNU-1 guidelines to identify patients with VAP. Definitions for VAP used in the literature are varied and may differ from ours. ${ }^{16}$ Our study may not have been sufficiently powered to detect true differences in risk given the number of therapies analyzed, although to our knowledge, this is the largest cohort of brain-injured pediatric patients with VAP reported to date. This was a single center study and results, particularly the antibiogram, may be specific to our geographic region and patient population and may not be representative of other settings.

\section{Conclusion}

VAP is a common occurrence among pediatric patients with moderate-to-severe TBI, particularly among those who are treated with therapies for intracranial hypertension that suppress cough and movement. VAP is associated with prolonged stay and functional morbidity at hospital discharge. While therapies designed to mitigate ICP may not necessarily be causally related to the development of VAP, they can serve as an indicator of patients at higher risk. Clinicians should have a high index of suspicion for VAP early in the course of TBI patients treated with neuromuscular blockade, cooling to euthermia, and barbiturates. In our population, the majority of pathogens were not resistant, and narrow-spectrum early empiric antibiotic coverage, but not prophylaxis, is appropriate in intubated pediatric TBI patients suspected of having pneumonia. Early narrowspectrum empiric therapy in any intubated pediatric TBI patient requiring neuromuscular blockade, cooling to euthermia, and barbiturate coma should be considered even in the absence of symptoms of VAP.

\section{Acknowledgments}

We would like to acknowledge Primary Children's Hospital Trauma Program and Primary Children's Hospital Department of Pharmacy, particularly Chanelle Stidham, PharmD, for their invaluable contributions to this manuscript.

Dr. Hamele is an active duty military officer. The views expressed in this article are those of the authors and do not reflect the official policy or position of the Department of the Army, Department of Defense, or the United States government.

\section{Author Disclosure Statement}

Tellen D. Bennett is supported by NICHD K23 HD074620. For the other authors, no competing financial interests exist.

\section{References}

1. Centers for Disease Control and Prevention. (2006). TBI By Age Group:14-15. Available at: www.cdc.gov/traumaticbraininjury/pdf/ tbi_blue_book_age.pdf. Accessed February 25, 2015.

2. Hutchison, J.S., Ward, R.E., Lacroix, J., Hébert, P.C., Barnes, M.A., Bohn, D.J., Dirks, P.B., Doucette, S., Fergusson, D., Gottesman, R., Joffe, A.R., Kirpalani, H.M., Meyer, P.G., Morris, K.P., Moher, D., Singh, R.N., and Skippen, P.W.; Hypothermia Pediatric Head Injury Trial Investigators and the Canadian Critical Care Trials Group. (2008).Hypothermia therapy after traumatic brain injury in children. N. Engl. J. Med. 358, 2447-2456.

3. Adelson, P.D., Wisniewski, S.R., Beca, J., Brown, S., Bell, M., Muiselaar, J., Okada, P., Beers, S., Balasubramani, G., and Hirtz, D. (2013). Comparison of hypothermia and normothermia after severe traumatic brain injury in children (Cool Kids): a phase 3, randomised controlled trial. Lancet Neurol. 12, 546-553.
4. Adelson, P.D., Ragheb, J., Muizelaar, J.P., Kanev, P., Brockmeyer, D., Beers, S., Brown, S., Cassidy, L., Chang, Y., and Levin, H. (2005). Phase II clinical trial of moderate hypothermia after severe traumatic brain injury in children. Neurosurgery 56, 740-754.

5. Alharfi, I.M., Stewart, T.C., Helali, I.A., Daoud, H., and Fraser, D.D. (2014). Infections in pediatric severe TBI. J. Neurotrauma 31, 452-458.

6. Gupta, S., Boville, B.M., Blanton, R., Lukasiewicz, G., Wincek, J., Chunjong, B., and Forbes, M. (2015). A multicentered prospective analysis of diagnosis, risk factors, and outcomes associated with pediatric ventilator-associated pneumonia. Pediatr. Crit. Care Med. 16. e65-e73.

7. Casado, R.J., de Mello, M.J., de Aragão, R.C., de Albuquerque Mde. F., and Correia, J.B. (2011). Incidence and risk factors for health careassociated pneumonia in a pediatric intensive care unit. Crit, Care Med. 39, 1968-1973.

8. Bigham, M.T., Amato, R., Bondurrant, P., Fridriksson, J., Krawczewski, C., Rakke, J., Ryckman, S., Schwartz, S., Shaw, J., Wells, D., and Brill, R. (2009). Ventilator-associated pneumonia in the pediatric intensive care unit: characterizing the problem and implementing a sustainable solution. J. Pediatr. 154, 582-587.e2.

9. Srinivasan, R., Asselin, J., Gildengorin, G., Wiener-Kronish, J., and Flori, H.R. (2009). A prospective study of ventilator-associated pneumonia in children. Pediatrics $123,1108-1115$.

10. Taira, B.R., Fenton, K.E., Lee, T.K., Meng, H., McCormack, J., Huang, E., Singer, A., and Scriven, R. (2009). Ventilator-associated pneumonia in pediatric trauma patients. Pediatr. Crit. Care Med. 10, 491-494.

11. Elward, A.M, Warren, D.K., and Fraser, V.J. (2002). Ventilatorassociated pneumonia in pediatric intensive care unit patients: risk factors and outcomes. Pediatrics 109, 758-764.

12. Al-Dorzi, H.M., El-Saed, A., Rishu, A.H., Balkhy, H.H., Memish, Z.A., and Arabi, Y.M. (2012). The results of a 6-year epidemiologic surveillance for ventilator-associated pneumonia at a tertiary care intensive care unit in Saudi Arabia. Am. J. Infect. Control 40, 794-799.

13. Almuneef, M., Memish, Z.A., Balkhy, H.H., Alalem, H., and Abutaleb, A. (2004). Ventilator-associated pneumonia in a pediatric intensive care unit in Saudi Arabia: a 30-month prospective surveillance. Infect. Control Hosp. Epidemiol. 25, 753-758.

14. Lepelletier, D., Roquilly, A., Demeure dit latte D, Mahe, P., Loutrel, O., Champin, P., Corvec, S., Naux, E., Pinaud, M., Lejus, C., and Asehnoune, K. (2010). Retrospective analysis of the risk factors and pathogens associated with early-onset ventilator-associated pneumonia in surgicalICU head-trauma patients. J. Neurosurg. Anesthesiol. 22, 32-37.

15. Fayon, M.J., Tucci, M., Lacroix, J., Farrell, C., Gauthier, M., Lafleur, L., and Nadeau, D. (1997). Nosocomial pneumonia and tracheitis in a pediatric intensive care unit: a prospective study. Am. J. Respir. Crit. Care Med. 155, 162-169.

16. Bradley, J.S. (2010). Considerations unique to pediatrics for clinical trial design in hospital-acquired pneumonia and ventilator-associated pneumonia. Clin. Infect. Dis. 51 Suppl 1(Suppl 1), S136-S143.

17. Acosta-Escribano, J., Fernández-Vivas, M., Grau Carmona, T., Caturla-Such, J., Garcia-Martinez, M., Menendez-Mainer, A., SoleraSuarez, M., and Sanchez-Payá, J. (2010). Gastric versus transpyloric feeding in severe traumatic brain injury: a prospective, randomized trial. Intensive Care Med. 36, 1532-1539.

18. Bradley, J.S., Byington, C.L., Shah, S.S., Alverson, B., Carter, E., Harrison, C., Kaplan, S., Mace, S., McCracken, G., Moore, M., St. Peter, S., Stockwell, J., and Swanson, J. (2011). The management of community-acquired pneumonia in infants and children older than 3 months of age: clinical practice guidelines by the Pediatric Infectious Diseases Society and the Infectious Diseases Society of America. Clin. Infect. Dis. 53, e25-e76.

19. Maas, A.I.R., Hukkelhoven, C.W.P.M., Marshall, L.F., and Steyerberg, E.W. (2005). Prediction of outcome in traumatic brain injury with computed tomographic characteristics: a comparison between the computed tomographic classification and combinations of computed tomographic predictors. Neurosurgery 57, 1173-1182.

20. Liesemer, K., Riva-Cambrin, J., Bennett, K.S., Bratton, S.L., Tran, H., Metzger, R.R., and Bennett, T.D. (2014). Use of Rotterdam CT scores for mortality risk stratification in children with traumatic brain injury. Pediatr. Crit. Care Med. 15, 554-562.

21. Kochanek, P.M., Carney, N., Adelson, P.D., Ashwal, S., Bell, M.J., Bratton, S., Carson, S., Chesnut, R.M., Ghajar, J., Goldstein, B., Grant, G.A., Kissoon, N., Peterson, K., Selden, N.R., Tasker, R.C., Tong, K.A., Vavilala, M.S., Wainwright, M.S., and Warden, C.R. (2012). Guidelines for the acute medical management of severe traumatic 
brain injury in infants, children, and adolescents-second edition. Pediatr. Crit. Care Med. Suppl 1, S1-82.

22. Clark, T.G., Bradburn, M.J., Love, S.B., and Altman, D.G. (2003). Survival analysis part IV: further concepts and methods in survival analysis. Br. J. Cancer 89, 781-786.

23. Therneau, T.C.C. (2014). Using Time Dependent Covariates and Time Dependent Coefficients in the Cox Model. Survival - R package version 2. 37.

24. Labenne, M., Poyart, C., Rambaud, C., Goldfarb, B., Pron, B., Jouvet, P., Delamare, C., Sebag, G., and Hubert, P. (1999). Blind protected specimen brush and bronchoalveolar lavage in ventilated children. Crit. Care Med. 27, 2537-2543.

25. Seguin, P., Laviolle, B., Dahyot-Fizelier, C., Dumont, R., Veber, B., Gergaud. S., Asehnoune, K., Mimoz, O., Donnio, P.Y., Bellissant, E., and Malledant, Y. (2014). Effect of oropharyngeal povidone-iodine preventive oral care on ventilator-associated pneumonia in severely brain-injured or cerebral hemorrhage patients: a multicenter, randomized controlled trial. Crit. Care Med. 42, 1-8.

26. Pelosi, P., Barassi, A., Severgnini, P., Gomiero, B., Finazzi, S., Merlini, G., d'Eril, G.M., Chiaranda, M., and Niederman, M.S. (2008). Prognostic role of clinical and laboratory criteria to identify early ventilator-associated pneumonia in brain injury. Chest 134, 101-108.

27. Patel, C.B., Gillespie, T.L., Goslar, P.W., Sindhwani, M., and Petersen, S.R. (2011). Trauma-associated pneumonia in adult ventilated patients. Am. J. Surg. 202, 66-70.

28. Sirvent, J.M., Torres, A., El-Ebiary, M., Castro, P., De Batlle, J., and Bonet, A. (1997). Protective effect of intravenously administered cefuroxime against nosocomial pneumonia in patients with structural coma. Am. J. Respir. Crit. Care Med. 155, 1729-1734.

29. Decelle, L., Thys, F., Zech, F., and Verschuren, F. (2011). Ventilationassociated pneumonia after intubation in the prehospital or the emergency unit. Eur. J. Emerg. Med. 20, 61-63.
30. Das, M., Mohapatra, S., and Mohapatra, S.S. (2012). New perspectives on central and peripheral immune responses to acute traumatic brain injury. J. Neuroinflammation. 9, 236.

31. Eberhardt, K.E.W., Thimm, B.M., Spring, A., and Maskos, W.R. (1992). Dose-dependent rate of nosocomial pulmonary infection in mechanically ventilated patients with brain edemia receiving barbiturates - a prospective case study. Infection. 20, 12-18.

32. Sato, M., Tanaka, S., Suzuki, K., Kohama, A., and Fujii, C. (1989). Complications associated with barbiturate therapy. Resuscitation. 17, 233-241.

33. Stover, J.F. and Stocker, R. (1998). Barbiturate coma may promote reversible bone marrow suppression in patients with severe isolated traumatic brain injury. Eur. J. Clin. Pharmacol. 54, 529-534.

34. Bratton, S.L., Chestnut, R.M., Ghajar, J., McConnell Hammond, F.F., Harris, O.A., Hartl, R., Manley, G.T., Nemecek, A., Newell, D.W., Rosenthal, G., Schouten, J., Shutter, L., Timmons, S.D., Ullman, J.S., Videtta, W., Wilberger, J.E., and Wright, D.W. (2007). Guidelines for the Management of Severe Traumatic Brain Injury 3rd Edition. J. Neurosurg. 24, Suppl (212), S1-S106.

Address correspondence to:

Maj. Mitchell Hamele, MD

United States Army Medical Corps

Department of Pediatrics

University of Utah

295 Chipeta Way, PO Box 581289

Salt Lake City, UT 84158

E-mail: mitchell.hamele@hsc.utah.edu

or

mitchell.hamele@us.army.mil 